\title{
PREVENTION OF HALO FORMATION IN HIGH BRIGHTNESS BEAMS
}

\author{
Yuri K. Batygin, \\ The Institute of Physical and Chemical Research (RIKEN), Saitama, 351-01, Japan
}

\section{Abstract}

Four vanes quadrupole structure with duodecapole field component is analyzed to suppress emittance growth and halo formation in high brightness beams. Beam profile in such a structure has to be close to square instead of conventional circle cross section. Adiabatic change of electrode shapes along the channel results in transformation of initially nonuniform beam into the beam, matched with the channel with pure quadrupole field. Results of prevention of halo formation in the bright beam with phase space density of $1.5 \mathrm{~A} /(\pi \mathrm{cm} \mathrm{mrad})$ are given.

\section{INTRODUCTION}

Emittance conservation of a high brightness particle beam is an issue for existing and future high intensity accelerator projects. Nonuniform space charge dominated beam is mismatched with linear focusing channel. It results in beam emittance growth and halo formation (see Fig.1). In Refs. [1, 2] it was shown that incorporation of a duodecapole component in pure quadrupole alternatinggradient structure provides better matching of the beam with the transport channel. Special case is a four-vanes structure, where shape of electrodes is modified to create multipole field distribution [3]. Potential of an uniform four vanes structure is given by

$$
\mathrm{U}(\mathrm{r}, \varphi, \mathrm{t})=\left(\frac{\mathrm{G}_{2}}{2} \mathrm{r}^{2} \cos 2 \varphi+\frac{\mathrm{G}_{6}}{6} \mathrm{r}^{6} \cos 6 \varphi\right) \sin \omega_{\mathrm{o}} \mathrm{t},
$$

where $G_{2}$ is a quadrupole gradient, $G_{6}$ is a duodecapole component and $\omega_{0}=2 \pi \mathrm{c} / \lambda$ is an operational frequency. Averaging of equations of motion of particle with mass $\mathrm{m}_{\mathrm{o}}$, charge $\mathrm{q}$ and energy $\gamma$ gives an effective scalar potential of the structure, which describes smoothed particle trajectories $[2,3]$ :

$$
\mathrm{U}_{\mathrm{ext}}(\mathrm{r}, \varphi)=\frac{\mathrm{m}_{\mathrm{o}} \mathrm{c}^{2}}{\mathrm{q}} \frac{\mu_{\mathrm{o}}^{2}}{\lambda^{2}}\left[\frac{\mathrm{r}^{2}}{2}+\zeta \mathrm{r}^{6} \cos 4 \varphi+\frac{\zeta^{2}}{2} \mathrm{r}^{10}\right]
$$

where $\mu_{\mathrm{o}}$ is a smoothed transverse oscillation frequency and $\zeta$ is a ratio of field components:

$$
\mu_{\mathrm{o}}=\frac{\mathrm{q} \mathrm{G}_{2} \lambda^{2}}{\sqrt{8 \gamma} \pi \mathrm{m}_{\mathrm{o}} \mathrm{c}^{2}}, \quad \zeta=\frac{\mathrm{G}_{6}}{\mathrm{G}_{2}} .
$$

The effective potential (2) is an axial - nonsymmetrtic and a highly nonlinear radius function. Equipotential lines $\mathrm{U}_{\text {ext }}(\mathrm{r}, \varphi)=\mathrm{C}$ are circles near axis and are transformed to a $45^{\circ}$ skewed square far from the axis [2].
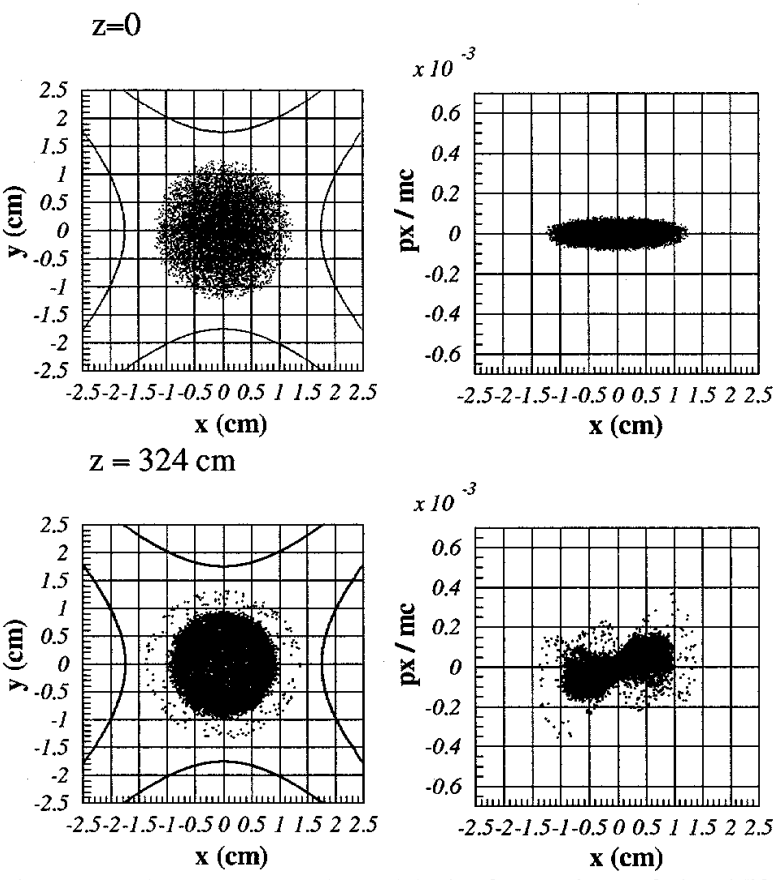

Fig. 1. Emittance growth and halo formation of the 150 $\mathrm{keV}, 100 \mathrm{~mA}, 0.06 \pi \mathrm{cm}$ mrad nonuniform proton beam in a four vanes quadrupole structure.

Beam emittance is conserved, if beam is matched with the channel. Finding matched conditions for the beam requires solution of self-consistent problem for beam distribution function in phase space. Self consistent particle distribution creates potential, in which particle motion maintains this distribution.

\section{SELF-CONSISTENT PARTICLE DISTRIBUTION IN A CONTINUOUS FOCUSING CHANNEL}

General approach to find a self-consistent distribution function in uniform focusing channel is to represent it as a function of Hamiltonian [4]

$$
f=f(H), \quad H=\frac{p_{x}^{2}+p_{y}^{2}}{2 m_{o} \gamma}+q U_{e x t}+q \frac{U_{b}}{\gamma^{2}},
$$

where $\mathrm{U}_{b}$ is a space charge potential of the beam. Convenient way is to use an exponential function $\mathrm{f}=\mathrm{f}_{\mathrm{o}} \exp \left(-\mathrm{H} / \mathrm{H}_{\mathrm{o}}\right)$. Distribution function contains two unknown constants $\mathrm{H}_{\mathrm{O}}, \mathrm{f}_{\mathrm{O}}$, which can be expressed through beam parameters. Root-mean-square (rms) beam emittance $\varepsilon$ is defined via beam radius $R=2 \sqrt{\left\langle x^{2}\right\rangle}$ and rms beam size in phase space $\mathrm{p}_{\mathrm{o}}=2 \sqrt{\left\langle\mathrm{p}_{\mathrm{X}}^{2}\right\rangle}$ :

$$
\varepsilon=\frac{4}{m_{0} c} \sqrt{\left\langle x^{2}\right\rangle\left\langle p_{x}^{2}\right\rangle}=\frac{R p_{o}}{m_{o} c} .
$$


Comparing definition of distribution function and eqs.(4), (5), the value of $\mathrm{H}_{\mathrm{O}}$ is given by:

$$
\mathrm{H}_{\mathrm{o}}=\frac{\mathrm{p}_{\mathrm{o}}^{2}}{4 \mathrm{~m}_{\mathrm{o}} \gamma}=\frac{\mathrm{m}_{\mathrm{o}} \mathrm{c}^{2}}{4 \gamma}\left(\frac{\varepsilon}{\mathrm{R}}\right)^{2} .
$$

Space charge density of the beam is expressed through beam distribution function after integration over particle momentum:

$$
\rho=q \int_{-\infty}^{\infty} \int_{-\infty}^{\infty} f d p_{x} d p_{y}=\rho_{o} \exp \left(-q \frac{U_{e x t}+\gamma^{-2} U_{b}}{H_{O}}\right),
$$

where $\rho_{o}=2 \pi q \gamma m_{o} f_{o} H_{o}$ is a value of space charge particle density in the center of the beam. Value of $\rho_{0}$ is unknown at this point due to unknown self potential of the beam $\mathrm{U}_{\mathrm{b}}$. Typical particle distribution of a laboratory beam has a maximum density in the center of the beam, which drops toward beam periphery. Let us introduce an average value of space charge density of the beam $\bar{\rho}=I /\left(\beta c \pi R^{2}\right)$, where $I$ is a beam current and $\beta$ is a particle velocity. In general case space charge density in the center of the beam $\rho_{0}$ differs from the average value of space charge density $\overline{\boldsymbol{\rho}}$ as a factor of $k$, where parameter $\mathrm{k}=1$ for uniform distribution $\rho(\mathrm{r})=\rho_{\mathrm{o}}$ and $\mathrm{k}=2$ for Gaussian distribution $\rho(r)=\rho_{o} \exp \left(-2 r^{2} / R^{2}\right)$. Taking into account adopted relationship $\rho_{o}=k \bar{\rho}$, the value of $f_{o}$ is expressed as follow:

$$
\mathrm{f}_{\mathrm{o}}=\mathrm{k} \frac{2 \mathrm{I}}{\pi^{2} \beta \mathrm{qm} \mathrm{m}_{\mathrm{o}}^{2} \varepsilon^{2}} .
$$

Substitution of the distribution function into Poisson's equation gives a nonlinear equation for unknown space charge potential of the beam $U_{b}$. After solution of the Poisson's equation, one can find the self-consistent particle distribution, which will be maintained in the focusing channel. Let us introduce dimensionless variables:

$$
V_{e x t}=\frac{q U_{e x t}}{H_{O}}, V_{b}=\frac{q U_{b}}{H_{O}}, \quad \xi=\frac{r}{a},
$$

where a is a channel radius. In new variables, Poisson's equation in cylindrical polar coordinates is

$$
\frac{1}{\xi} \frac{\partial}{\partial \xi}\left(\xi \frac{\partial \mathrm{V}_{\mathrm{b}}}{\partial \xi}\right)+\frac{\partial^{2} \mathrm{~V}_{\mathrm{b}}}{\xi^{2} \partial \varphi^{2}}=-8 \mathrm{~kb}\left(\gamma \frac{\mathrm{a}}{\mathrm{R}}\right)^{2} \exp \left(-\mathrm{V}_{\mathrm{ext}}-\frac{\mathrm{V}_{\mathrm{b}}}{\gamma^{2}}\right),(10)
$$

where $b$ is a dimensionless value of beam brightness:

$$
\mathrm{b}=\frac{2}{\beta \gamma} \frac{\mathrm{I}}{\mathrm{I}_{\mathrm{c}}}\left(\frac{\mathrm{R}}{\varepsilon}\right)^{2},
$$

and $I_{c}=4 \pi \varepsilon_{0} m_{o} c^{3} / q=3.13 \cdot 10^{7}(A / Z)$ Amp is a characteristic value of beam current.

Unknown space charge potential of the beam can be expressed as Fourier-Bessel series

$$
\mathrm{V}_{\mathrm{b}}=\mathrm{V}_{\mathrm{O}}+\sum_{\mathrm{n}=0}^{\infty} \sum_{\mathrm{m}=1}^{\infty} \mathrm{J}_{\mathrm{n}}\left(v_{\mathrm{nm}} \xi\right)\left(\mathrm{A}_{\mathrm{nm}} \cos \mathrm{n} \varphi+\mathrm{B}_{\mathrm{nm}} \sin \mathrm{n} \varphi\right)
$$

where $\mathrm{J}_{\mathrm{n}}(\mathrm{x})$ is a Bessel function and $v_{\mathrm{nm}}$ is a m-th root of the equation $\mathrm{J}_{\mathrm{n}}(\mathrm{x})=0$. Constant $\mathrm{V}_{\mathrm{o}}=-\mathrm{bk} \gamma^{2}$ is defined in such a way, that total potential of the structure vanishes at the axis $V_{\text {ext }}(0, \varphi)+V_{b}(0, \varphi) \gamma^{-2}=0$.

To find an approximate solution of Poisson's equation, let us take the first term in the near-axis expansion of exponential function $\exp \left(-\mathrm{V}_{\mathrm{ext}}-\mathrm{V}_{\mathrm{b}} \gamma^{-2}\right) \approx 1-\mathrm{V}_{\mathrm{ext}}-\mathrm{V}_{\mathrm{b}} \gamma^{-2}$. Poisson's equation becomes:

$$
\begin{gathered}
\sum_{\mathrm{n}=0}^{\infty} \sum_{m=1}^{\infty}\left[1+\frac{v_{\mathrm{nm}}^{2}}{8 \mathrm{~kb}}\left(\frac{\mathrm{R}}{\mathrm{a}}\right)^{2}\right] \mathrm{J}_{\mathrm{n}}\left(v_{\mathrm{nm}} \xi\right)\left(\mathrm{A}_{\mathrm{nm}} \cos \mathrm{n} \varphi+\mathrm{B}_{\mathrm{nm}} \sin \mathrm{n} \varphi\right)= \\
=\left(1-\mathrm{V}_{\text {ext }}\right) \gamma^{2}-\mathrm{V}_{\mathrm{o}}
\end{gathered}
$$

Space charge dominated beam transport is achieved, if $\mathrm{b}>>1$. It gives the possibility to simplify approximation of Poisson's equation (13). Expression in square brackets in equation (13) can be taken as a constant, close to unit:

$$
1+\frac{v_{\mathrm{nm}}^{2}}{8 \mathrm{~kb}}\left(\frac{\mathrm{R}}{\mathrm{a}}\right)^{2} \approx 1+\delta, \quad \delta=\frac{1}{\mathrm{bk}} \ll<1 .
$$

With that approximation, self-consistent space charge dominated beam potential is:

$$
\mathrm{V}_{\mathrm{b}}=-\frac{\gamma^{2}}{1+\delta} \mathrm{V}_{\mathrm{ext}}
$$

Second approximation to the self-consistent potential is given by holing one more term in expansion of exponential function $\exp \left(-\mathrm{V}_{\mathrm{ext}}-\mathrm{V}_{\mathrm{b}} \gamma^{-2}\right) \approx 1-\mathrm{V}_{\mathrm{ext}}-\mathrm{V}_{\mathrm{b}} \gamma^{-2}+$ $+0.5\left(\mathrm{~V}_{\mathrm{ext}}-\mathrm{V}_{\mathrm{b}} \gamma^{-2}\right)^{2}$ :

$$
\mathrm{V}_{\mathrm{b}}=\gamma^{2}\left[1+\delta-\mathrm{V}_{\mathrm{ext}}-\sqrt{\left(1+\delta-\mathrm{V}_{\mathrm{ext}}\right)^{2}-\mathrm{V}_{\mathrm{ext}}\left(\mathrm{V}_{\mathrm{ext}}-2\right)}\right] .
$$

From eqs. (15), (16) it follows, that space charge potential of a high brightness matched beam always tends to the same distribution as an external focusing potential with opposite sign, regardless the focusing field is applied. With increasing of beam brightness, exact solution of eq. (10) becomes close to eq.(15). In the extreme case of infinitely high bright beam, space charge potential of the beam completely compensates for focusing field $\mathrm{V}_{\mathrm{b}}=-\gamma^{2} \mathrm{~V}_{\text {ext }}$.

Self consistent space charge distribution of matched beam in the channel with potential (2) is given by

$$
\rho_{\mathrm{b}}=\frac{\varepsilon_{\mathrm{o}}}{1+\delta} \gamma^{2} \Delta \mathrm{U}_{\mathrm{ext}}=\rho_{\mathrm{o}}\left(1+10 \zeta \mathrm{r}^{4} \cos 4 \varphi+25 \zeta^{2} \mathrm{r}^{8}\right) .
$$

In the limit of very high brightness beam $\delta \rightarrow 0$ space charge density of the matched beam (17) does not depend on beam brightness. Particle distribution (17) has a 4folded symmetry. Every $45^{\circ}$ variation of azimuth angle results in change of particle distribution from decreasing to increasing function of radius and vice versa.

Realistic beam distribution has monotonically decreasing density function of radius. Good approximation to realistic beam is a parabolic distribution 
in phase space [2, 3], which gives projection in real space as $\rho(r)=\rho_{o}\left[1-0.5(r / R)^{2}\right]^{2}$, close to truncated Gaussian distribution . In Refs. [2,3] expressions for required values of quadrupole gradient and duodecapole components to provide approximate matching of such a beam with the channel were found:

$$
\begin{aligned}
& \mathrm{G}_{2}=\frac{\sqrt{8} \pi \mathrm{m}_{\mathrm{o}} \mathrm{c}^{2}}{\mathrm{q} \lambda \mathrm{R}} \sqrt{\frac{\varepsilon^{2}}{\mathrm{R}^{2}}+\frac{3 \mathrm{I}}{\mathrm{I}_{\mathrm{c}} \beta \gamma}}, \\
& \mathrm{G}_{6}=-\frac{\mathrm{G}_{2}}{12 \beta \gamma \mathrm{R}^{4}} \frac{\mathrm{I}}{\mathrm{I}_{\mathrm{c}}}\left(\frac{\varepsilon^{2}}{\mathrm{R}^{2}}+\frac{3 \mathrm{I}}{\mathrm{I}_{\mathrm{c}} \beta \gamma}\right)^{-1} .
\end{aligned}
$$

To make the realistic beam distribution as close to matched beam distribution as possible, beam has to be truncated along equipotential lines. Therefore, beam boundaries have to be $45^{\circ}$ skewed square.

\section{RESULTS OF PARTICLE-IN-CELL SIMULATION}

Computer simulations using particle-in-cell code BEAMPATH [5] were done to verify the derived matched conditions of the beam. Nonlinear transformer with adiabatic change of duodecapole component was tested. If parameters of the structure are changed adiabatically, beam emittance is a constant of motion. One can expect modification of a nonuniform particle distribution into more uniform distribution in the structure, where higher order focusing field components drops gradually.

In Fig. 2 results of beam dynamics in quadrupole channel with gradual variation of a duodecapole component are presented. The value of quadrupole
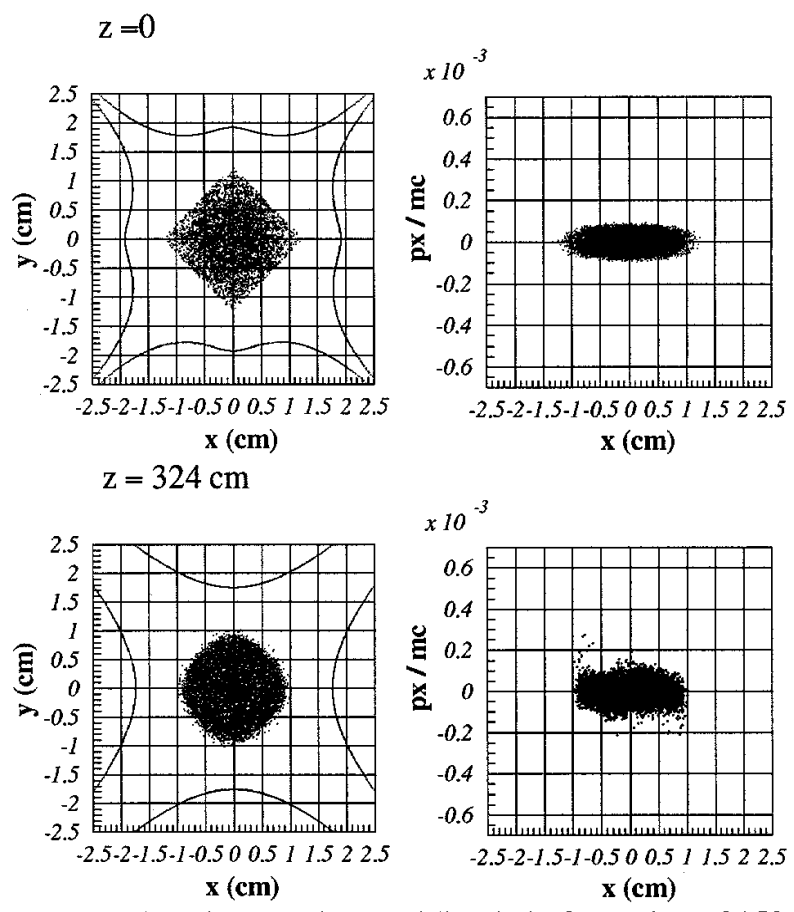

Fig. 2. Adiabatic matching avoiding halo formation of $150 \mathrm{keV}, 100 \mathrm{~mA}, 0.06 \pi \mathrm{cm}$ mrad proton beam in a nonlinear matching section. gradient $\mathrm{G}_{2}=50 \mathrm{kV} / \mathrm{cm}^{2}$ was kept constant along the channel. The duodecapole component was adiabatically changed from the value $\mathrm{G}_{6}=-1.9 \mathrm{kV} / \mathrm{cm}^{6}$ as required by matched conditions, to zero for the distance $\mathrm{L}=100 \mathrm{~cm}$ utilizing linear law $\mathrm{G}_{6}(\mathrm{z})=\mathrm{G}_{6}(1-\mathrm{z} / \mathrm{L})$. After nonlinear matching section $\mathrm{z}>\mathrm{L}$, the channel was a pure quadrupole and the beam was transported $224 \mathrm{~cm}$ more to check the results of transformation. As seen, beam profile in real space is modified from a square to a circular shape. Rms beam emittance growth in simulations, presented in Fig. 2 is $15 \%$, which is substantially smaller, than $50 \%$ emittance growth in a pure quadrupole channel, presented in Fig. 1. The final beam emittance and beam profile are matched without serious phase space portrait distortion. After transformation, the beam can be transported in a conventional structure with a linear focusing field.

\section{CONCLUSIONS}

Self consistent space charge potential of a high brightness beam is derived in case of arbitrary potential of continuos focusing channel. It is shown, that matched beam always tends to compensate for applied potential. Simple formula is given, which demonstrates the effect of shielding in case of arbitrary focusing potential. Four vanes quadrupole structure with a multipole component of the 6th order is analyzed to prevent space charge dominated beam emittance growth. In such a structure, the matched beam profile has to be close to square, instead of the conventional circle beam cross section. The adiabatic change of a nonlinear focusing field along a structure results in gradual transformation of an initially nonuniform beam distribution into a distribution matched with the linear focusing channel. Given analysis provides matched conditions for a non-uniform high brightness particle beam transport without beam halo formation.

\section{REFERENCES}

[1] Y. Batygin, Phys. Review E, 53, 5358, (1996).

[2] Y.Batygin, Phys. Review E, 54, 5673, (1996).

[3] Y.Batygin, A.Goto and Y.Yano, Proceedings of the 5th Europ. Part. Acc. Conference (EPAC96), Barcelona, 1236 (1996).

[4] I.M.Kapchinsky, Theory of Resonance Linear Accelerators, Harwood, Chur, 1986.

[5] Y. Batygin, Proceedings of the 3rd European Part. Acc. Conference (EPAC92), Berlin, 822 (1992). 